\title{
3D Metallic Plate Lens Antenna Based BEAMSPACE CHANNEL EsTIMATION TECHNIQUE FOR 5G MMWAVE MASSIVE MIMO SYSTEMS
}

\author{
Prosenjit Paul and Md Munjure Mowla \\ Department of Electronics and Telecommunication Engineering, \\ Rajshahi University of Engineering \& Technology, Rajshahi, Bangladesh.
}

\begin{abstract}
Beamspace channel estimation mechanism for massive MIMO (multiple input multiple output) antenna system presents a major process to compensate the $5 G$ spectrum challenges caused by the proliferation of information from mobile devices. However, this estimation is required to ensure the perfect channel state information (CSI) for lower amount of Radio Frequency $(R F)$ chains for each beam. In addition, phase shifter (PS) components used in this estimation need high power to select the beam in the desired direction. To overcome these limitations, in this work, we propose Regular Scanning Support Detection (RSSD) based channel estimation mechanism. Moreover, we utilise a 3D lens antenna array having metallic plate and a switch in our model which compensates the limitation of phase shifters. Simulation results show that the proposed RSSD based channel estimation surpasses traditional technique and SD based channel estimation even in lower SNR area which is highly desirable in the millimeter wave (mmWave) massive MIMO systems.
\end{abstract}

\section{KEYWORDS}

5G, Millimeter wave, Massive MIMO, Metallic plate lens antenna array, Beamspace Channel Estimation.

\section{INTRODUCTION}

It is highly expected that the fifth generation (5G) standard will support a massive number of wireless connections and will provide end to end high speed data rates. To fill up this demand, the mmWave system [1] has been considered as a pivotal technique in recent years because it holds up a wider channel bandwidth (BW) [2]. Nevertheless, it is very challenging task to implement mmWave in a massive MIMO system (mMIMO). In general, a mMIMO system utilizes a single radio frequency (RF) chain for a single antenna. The total amount of resulting antennas needs high number of RF chains and consumes high energy [2]. As a result, the mmWave mMIMO system faces high hardware complexity and wastage of energy. However, this limitation imposes challenges for next generation 5G applications. Beamspace channel estimation has been getting popular in mmWave MIMO systems which can utilize beam selection for minimizing RF chains without degrading obvious system loss [3]. The high absorption losses in mmWave bands results low effectual propagation paths where small number of beams possess uniquely. For that reason, a smaller number of necessary beams is required to reduce the size of mmWave mMIMO system as well as the number of necessary RF chains [4].

However, a base station requires collecting exact data of beamspace channel to select a ray which is not easy in real system, specifically at the time of limited amount of RF chains. Nowadays for solving this barrier, some techniques have been proposed on the basis of compressive sensing (CS) $[3,4,5]$. The main observation from these mechanisms is to take advantage of the scattering 
of mmWave channels in the directed area to accurately calculate the mmWave mMIMO channels of broad dimension. Nevertheless, hybrid precoding systems [6] exercise those mechanisms and the Phase Shifters (PS) are used to create the right angle to pass the information to the desired location as per user demand. On the other hand, lens antenna array is exercised instead of PS in beamspace MIMO systems and the formed beams are predetermined by restricted directions. In a beamspace MIMO system impartially exerting the present channel estimation technique, the exact acquisition of beams is very difficult [2]. 3D metallic plate lens antenna array is helpful to concentrating beams from several directions. This motivates us to estimate a channel using 3D metallic plate lens antenna array at the beamspace MIMO system which were not adequately characterized in earlier works.

In this research, we consider a new channel estimation technique with 3D metallic plate lens antenna array which saves energy in addition to focus beams. A signal transmits from precoder part will be twice in the presence of voltage doubler which power consuming is less relevant than its output. To detect the signal according to a column of the signal matrix, we propose an Regular Scanning Support Detection (RSSD) based channel estimation technique. Initially, entire channel estimation obstacles are divided by some alternate obstacles named as channel component. After that using the regular scanning the component support is detected along with fading action. Finally, channel component will be abolished from the entire beamspace channel estimation complication. For the next detection we also use this method. The nonzero ingredients of the infrequent beamspace channel are approximated after the detection of all support for channel component. Simulation outcomes describe that in low signal to noise ratio (SNR) region, the proposed RSSD based channel estimation exceeds conventional MIMO based and Support detection (SD) based channel estimation which is more desirable for mmWave systems [18].

The organization of the paper is as follows: Section II describes about beamspace MIMO system model. Section III sketches the beamspace channel estimation technique and the proposed RSSD based channel estimation mechanism. Simulation result is portrayed in section IV. In the end, Section V concludes the research with further directions.

\section{SYSTEM MODEL}

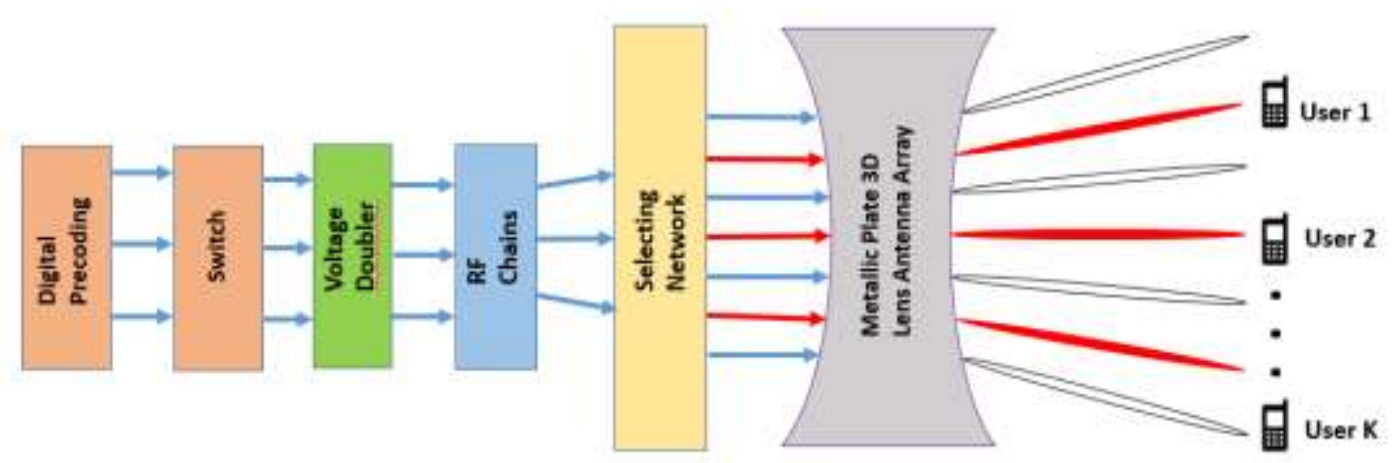

Figure 1. Structure of the system.

We consider a downlink mmWave massive MIMO system model which performs on time division duplexing (TDD) approach. The base station (BS) of this system model holds $N$ antennas as well as $N_{R F}$ RF chains to serve K users. The downlink signal power for this system model can be written as [7]:

$$
y^{d l}=H^{H} P s+n
$$


Where $P$ denotes precoding matrix, $n$ describes noise matrix, $s$ defines the signal vector and $H^{H}$ denotes the downlink channel matrix.

\section{1. mmWave Channel Model}

In this research, we use 3D lens antenna array to enhance beam estimation in both horizontal and vertical sparsity. Unlike traditional 2D channel model [2], [8] we adopt popular 3D SalehValenzuela channel model for mmWave communications [9]. The channel model can be expressed as:

$$
h_{k}=\sqrt{\frac{N}{L+1}} \sum_{i=0}^{L} \beta_{k}^{(i)} a\left(\psi_{k}^{(i)}\right)\left(\theta_{k}^{(i)}\right)=\sqrt{\frac{N}{L+1}} \sum_{i=0}^{L} C_{i}
$$

here line-of-sight (LoS) element for $k$-th user can be demonstrated (i.e. $i=0)$ as [10]:

$$
C_{0}=\beta_{k}^{(0)} a\left(\psi_{k}^{(0)} \theta_{k}^{(0)}\right) .
$$

where $\beta_{k}^{(0)}$ illustrates the complex gain and $\psi_{k}^{(0)} \theta_{k}^{(0)}$ denotes the spatial azimuth (elevation). Similarly, the Non-LOS (NLoS) components [10] can be characterized as $i^{\text {th }}$ NLoS components for $h_{k}$ having $k$-th user as [11]:

$$
C_{i}=\beta_{k}^{(i)} a\left(\psi_{k}^{(i)} \theta_{k}^{(i)}\right)
$$

where $L$ stands for the total amount of components [12].

The proposed system model uses 3D lens antenna array which scatters the beam in both horizontally and vertically. In this case, uniform planar array (UPA) is used to characterize the beam steering with a number of elements in both directions (i.e., $N_{1}$ for horizontally and $N_{2}$ for vertically). Therefore, the spatial azimuth for UPA can be written as [13]:

$$
a(\psi, \theta)=\frac{1}{\sqrt{N_{1}}}\left[e^{-j 2 \pi \psi u}\right] \otimes \frac{1}{\sqrt{N_{2}}}\left[e^{-j 2 \pi \theta t}\right]
$$

where $u \in I\left(N_{1}\right)$ and $t \in I\left(N_{2}\right)$.

The azimuth $\psi=\frac{d_{1}}{\lambda} \sin \tilde{\psi}$ and the elevation $\theta=\frac{d_{2}}{\lambda} \sin \tilde{\theta}$ where $\tilde{\psi}$ and $\tilde{\theta}$ are the physical azimuth as well as elevation, $\lambda$ is the wavelength of carrier [14] and $d_{1}, d_{2}$ are antenna spacing.

\subsection{MIMO System with lens}

Figure 1 depicts the system model of 3D metallic plate lens antenna utilizing the SalehValenzuela concept. A matrix is generated by the lens antenna array indicated by $V$ and can be written as:

$$
V=\left[a\left(u / N_{1}, t / N_{2}\right)\right]^{H}
$$

where $u / N_{1}$ and $t / N_{2}$ are the spatial azimuths and elevations defined by lens antenna array [16]. Now the system architecture for the beamspace MIMO system will be portrayed as [15]: 


$$
y^{D L}=H^{T} V^{T} P S+n
$$

\section{BEAMSPACE CHANNEL}

In this section, we first describe the beamspace channel estimation procedure. The estimation of the equation for channel selection will be described later. Then we will show the performance analysis of the proposed RSSD techniques and compare with the existing SD technique and conventional MIMO technique.

\subsection{Beamspace Channel Estimation}

In channel estimation technique, time division duplexing (TDD) system is performed by transmitting the pilot sequences toward base station over $S$ instants for every user. Here we partition $S$ instants in $M$ block and $\phi_{m}$ is the pilot matrix for $m-t h$ block.

Now, according to the channel reciprocity [17] theorem, the received uplink signal matrix can be represented as:

$$
y_{m}^{U L}=V H \phi_{m}+n_{m}=H \phi_{m}+N_{m}, \quad m=1,2, \ldots \ldots, M
$$

Where $N_{m}$ is the noise matrix in the $m^{\text {th }}$ block. At the time of pilot transmission, the base station could be exploited as an analog combiner $W_{m}$ to combine $y_{m}^{U L}$ and obtain final combination as:

$$
R_{m}=W_{m} y_{m}^{U L}=W_{m} H \phi_{m}+W_{m} N_{m}
$$

Eventually the known pilot matrix is multiplied in the left side to form the measurement matrix of beamspace channel which can be expressed as:

$$
Z_{m}=R_{m} \phi_{m}^{H}=W_{m} H+N_{m}^{e f f}
$$

where $N_{m}^{\text {eff }}$ is the effective noise matrix.

Therefore, while transmitting the pilot sequence, the measurement vector $\bar{z}_{k}$ can be obtained as:

$$
\bar{z}_{k}=\left[\begin{array}{c}
z_{1, k} \\
\cdot \\
\cdot \\
z_{M, k}
\end{array}\right]=\left[\begin{array}{c}
W_{1} \\
\cdot \\
\cdot \\
W_{M}
\end{array}\right] h_{k}+\left[\begin{array}{c}
n_{1, k} \\
\cdot \\
\cdot \\
n_{M, k}
\end{array}\right]=\bar{W} h_{k}+\overline{n_{k}}
$$

where $h_{k}$ is $k^{\text {th }}$ column of $H$ which can be solved by channel estimation algorithm. However, in low SNR region it is very difficult to reduce noise interference. In this stage, we propose the RSSD based channel estimation technique which enhances system performances. 


\subsection{Proposed RSSD based Channel Estimation}

Detecting strongest element from the channel component is the main concept of SD [15] based channel estimation technique. But this method does not produce a desired result in all sectors especially in the low SNR region. Instead of strong detection the proposed method detects the component as column wise. Consequently, the signal which contains less power cannot erase primarily, rather alternatively changing their positions in the matrix are counted. Detecting strong signal may not produce desired result all time, while regular scanning of beamspace signal ensures proper channel estimation. This is the main mechanism of the proposed RSSD based channel estimation.

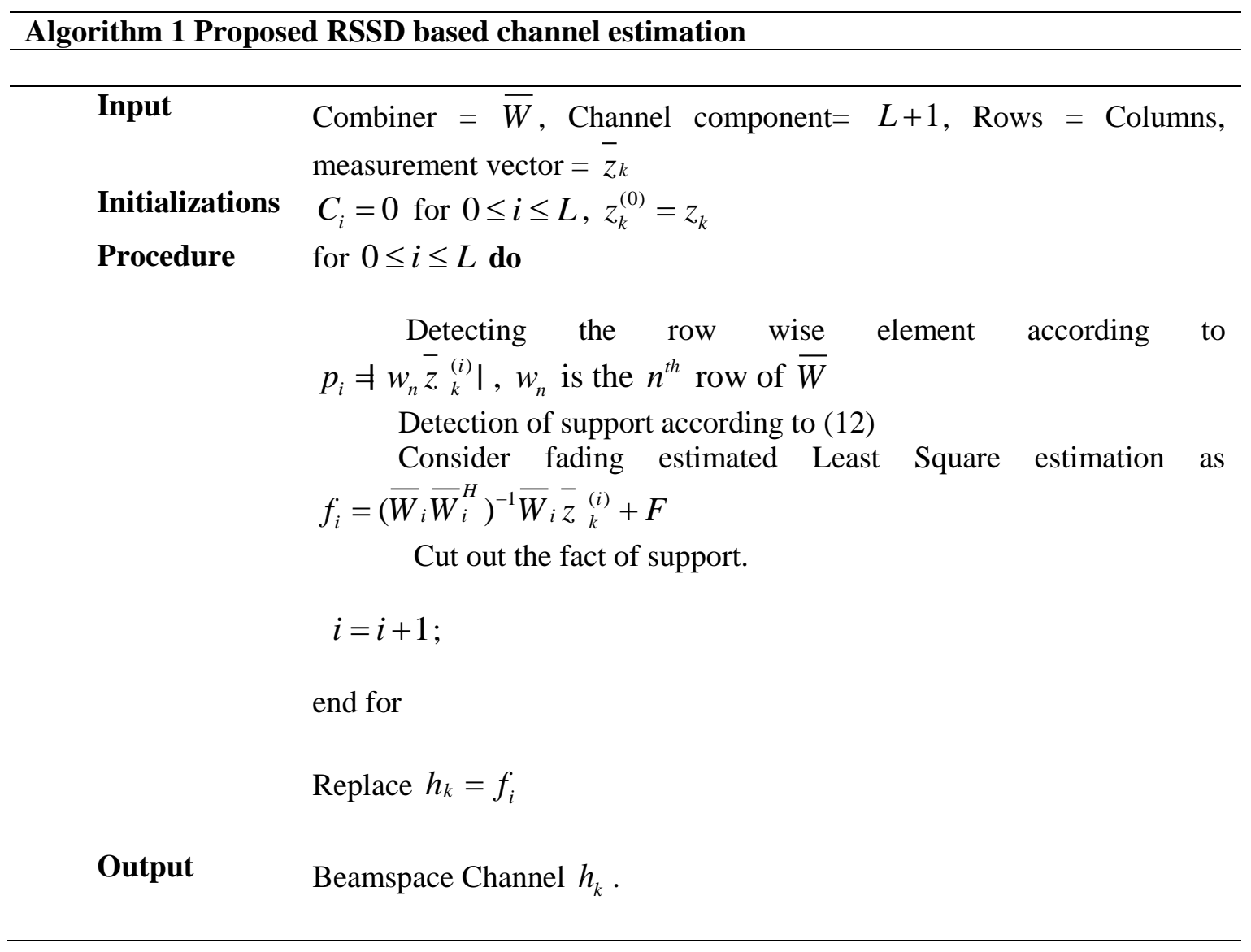

Now the support of sparse vector for $\tilde{c}_{i}=V c_{i}$ is described as:

$$
S_{i}=a-\frac{D}{2}, \ldots ., a+\frac{D-2}{2}
$$

where $a=$ ingredient of column as well as $D=$ all the amount of column. The term $\mathrm{F}$ can be represented as [7]:

$$
F=\frac{2 r}{\Omega} e^{-\frac{r^{2}}{\Omega}},
$$


where $\Omega=E\left[r^{2}\right]$ and $r$ presents random variable. Finally, influence of selecting matrix will eliminated as well as this procedure will run till the last channel has considered. Accumulating all the supports and removed them from one by one the total process will end. For this system model, the achievable sum rate (bit/sec/Hz), i.e., spectral efficiency $\mathrm{SE}$ ) can be written as:

$$
R=\log _{2}(1+\gamma)
$$

where $\gamma=\frac{\left|h_{k} \bar{W} P\right|^{2}}{\overline{n_{k}}+F}$ stands for signal to noise plus fading ratio. The energy efficiency of this model can be represented as [18]:

$$
\eta=\frac{R}{P_{T}}
$$

where total power consumption can be written as: $P_{T}=P_{t}+N_{R F} P_{R F}+N_{S W} P_{S W}$. In which, $\mathrm{P}_{\mathrm{t}}$, $P_{R F}$ and $P_{S W}$ denotes transmitted power, power consumption of RF chains, and power consumption of switch respectively. $\mathrm{N}_{\mathrm{RF}}$ and $\mathrm{N}_{\mathrm{SW}}$ represents the number of RF chains and switches respectively.

\section{Simulation RESUltS}

In this section, we analyse the performance of the proposed system model. A metallic plate lens antenna array is used to construct the BS. We consider $N=256$ antennas. This model works with $K=64,128$ users as well as $N_{R F}=64,128$ RF chains. Also, we consider the channel component $L=1$ and $L=3$ in case of LoS and NLoS, respectively. Now $d=0.5$ addresses as the antenna spacing which is suitable for the system. The carrier frequency of the system is considered as $28 \mathrm{GHz}$. The power consumption of the RF chain and switch is considered as 250 $\mathrm{mW}$ and $5 \mathrm{~mW}$, respectively [18]. The simulation parameters are shown in Table 1 .

Table 1: Simulation Parameters

\begin{tabular}{|c|c|}
\hline Parameters Name & Value \\
\hline Number of Transmit antennas $(N)$ & 256 \\
\hline Number of Users $(K)$ & 64,128 \\
\hline Number of paths per user $(L)$ & 1,3 \\
\hline Number of RF chain $\left(N_{R F}\right)$ & 64,128 \\
\hline Antenna spacing $(d)$ & $0.5 \mathrm{~m}$ \\
\hline Frequency & $28 \mathrm{GHz}$ \\
\hline Wavelength & $3 \mathrm{e} 8 / \mathrm{f}_{\mathrm{c}}$ \\
\hline Transmit Power $\left(P_{t}\right)$ & $1000(\mathrm{~mW})$ \\
\hline Power of RF chain $\left(P_{R F}\right)$ & $250(\mathrm{~mW})$ \\
\hline Power of switch $\left(P_{S W}\right)$ & $5(\mathrm{~mW})$ \\
\hline
\end{tabular}


International Journal of Wireless \& Mobile Networks (IJWMN) Vol. 13, No.1, February 2021

Fig. 2 represents the normalized mean square error (NMSE) analysis for the proposed model with exiting models. We depict the NMSE (dB) vs SNR (dB) graph for evaluating the performance. Figs. 2, 3, 4 sketch LoS, NLoS and fading effect for 64 users respectively.

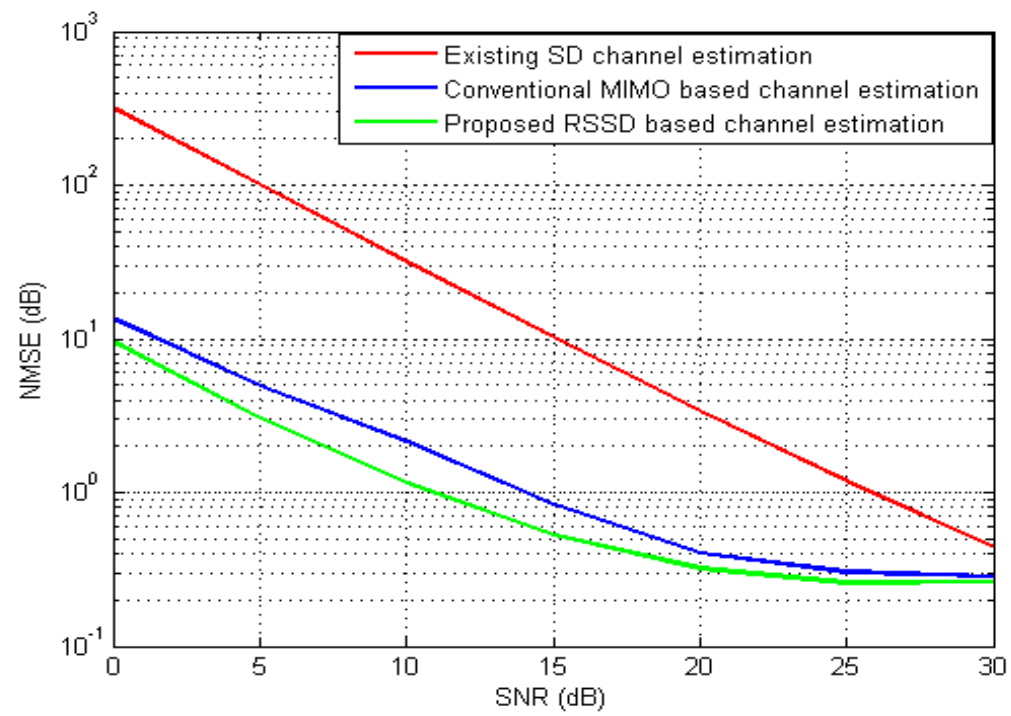

Figure 2. NMSE for LoS effect with 64 users.

Fig. 2 sketches that conventional MIMO based channel estimation has greater than $10^{1} \mathrm{~dB}$ NMSE rate and the current SD based channel estimation technique stands near $10^{3} \mathrm{~dB}$ NMSE rate.

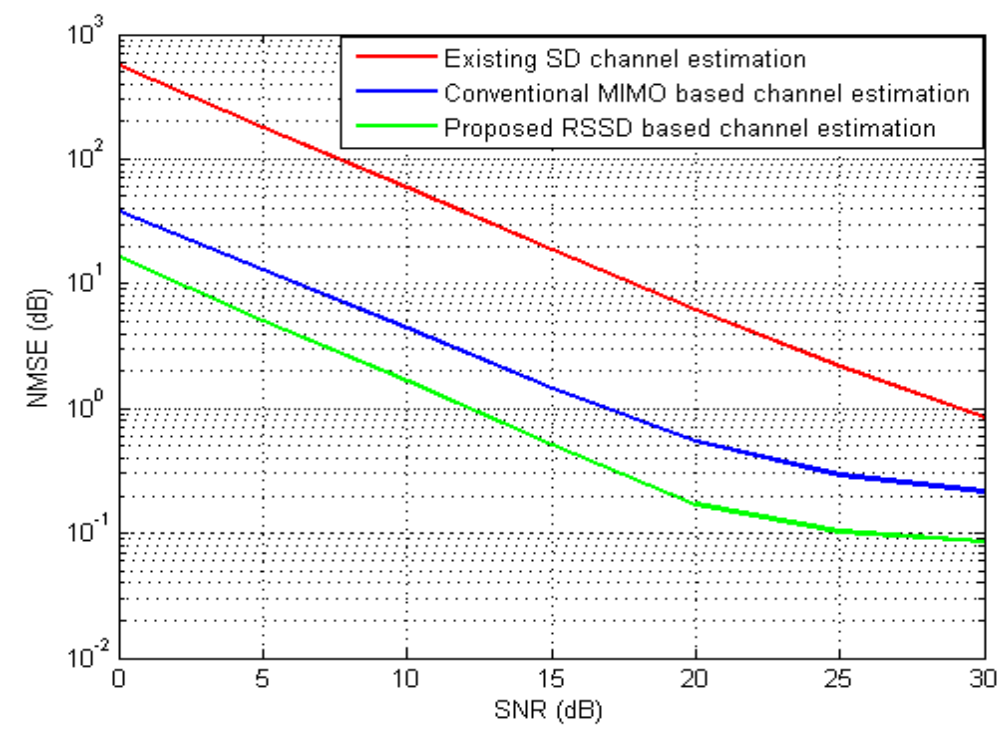

Figure 3. NMSE for NLoS effect with 64 users.

But in our proposed method we observe that the rate of NMSE is much lower than the other two techniques. 
International Journal of Wireless \& Mobile Networks (IJWMN) Vol. 13, No.1, February 2021

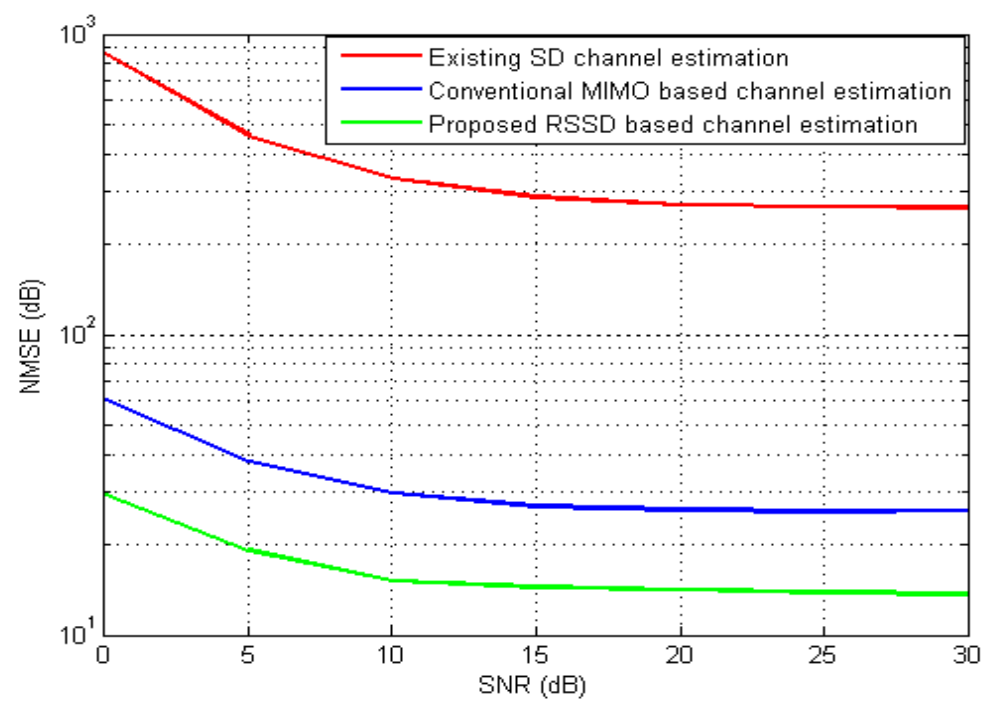

Figure 4. NMSE for fading effect with 64 users.

In Fig. 3 the NMSE rate shows for the NLoS effect. Here in low SNR region, the NMSE rate is same as the Fig. 2, but in high SNR region the NMSE rate becomes high compared with Fig. 2. Similarly, in Fig. 4 the current SD-based channel estimation draws a high NMSE in two (high and low) SNR areas. However, our proposed model portrays low NMSE rate in both low and high SNR region.

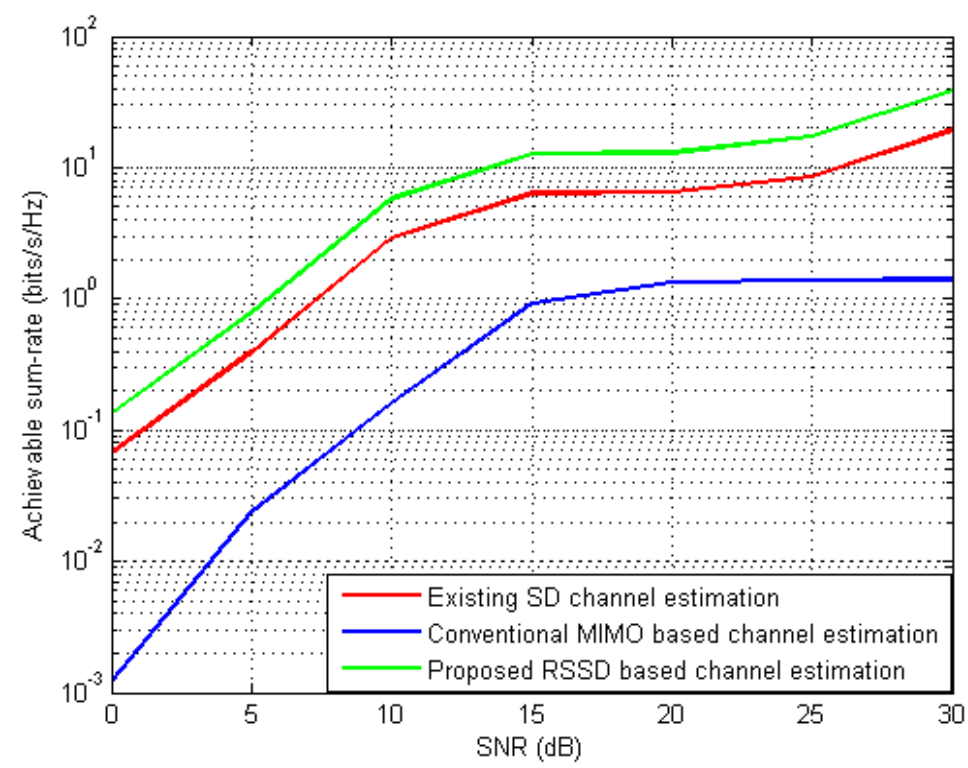

Figure 5. Achievable sum-rate (SE) for 64 users with LoS effect 


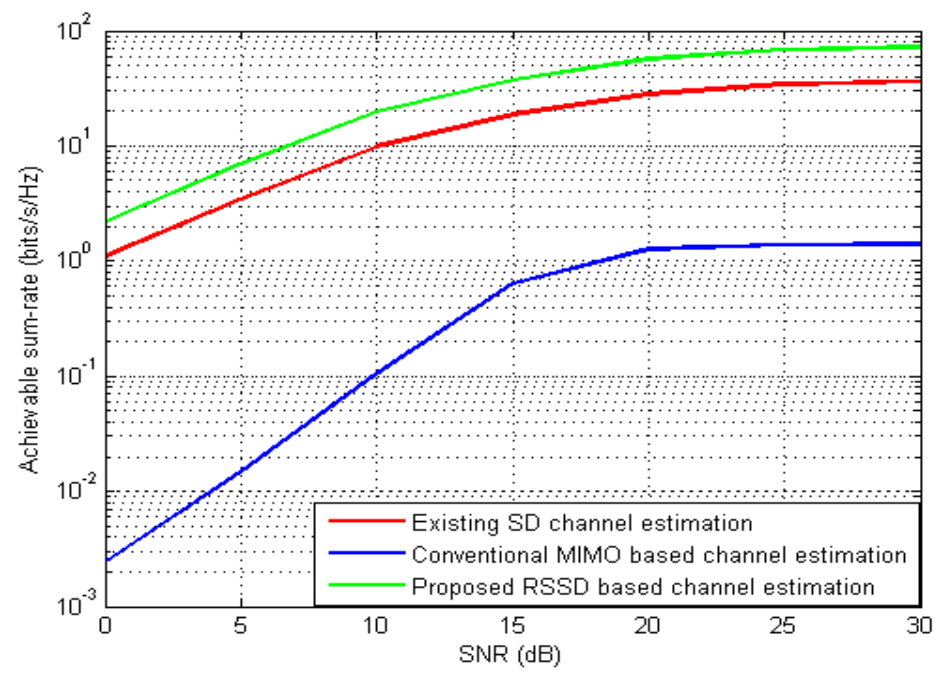

Figure 6. Achievable sum-rate (SE) for 64 users with NLoS effect.

In Fig. 6 the conventional MIMO based channel estimation gives the achievable sum-rate is near $10^{-3}$ in low SNR region and gradually increases with the increase SNR. The existing SD-based channel estimation has the sum rate near $10^{\circ}$ in lower SNR area. Nevertheless, our proposed technique gives us the better rate and also higher than the Fig. 5.

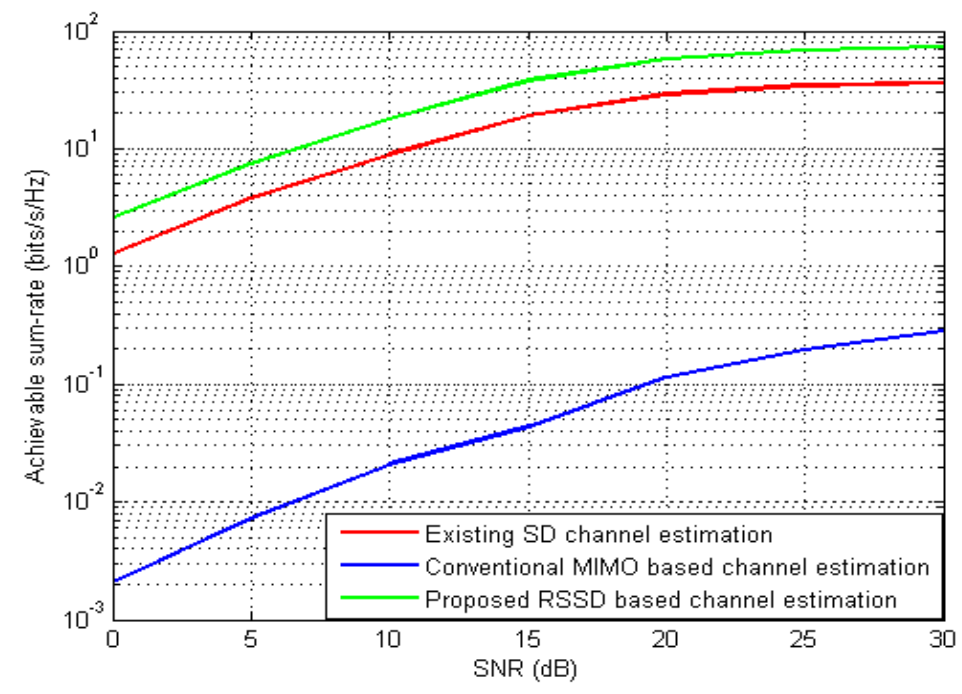

Figure 7. Achievable sum-rate (SE) for 64 users with fading effect.

Fig. 5 sketches the Spectral Efficiency (SE) for our proposed model. Here achievable sum rate vs SNR (dB) graph is drawn. Fig. 5, Fig. 6, and Fig. 7 shows the result of NMSE in case of LoS, NLoS, and fading effects. The conventional MIMO based channel estimation has SE (bits/s/Hz) near $10^{-3}$ in lower SNR area as well as in higher SNR area. Here we can see that it gradually increases in both regions. In existing SD-based channel estimation technique the Achievable sum-rate is near $10^{\circ}$. However, our proposed technique gives us the higher achievable sum-rate in two SNR area. In Fig. 7, the achievable sum-rate is shown for the fading effect. Here the 
conventional MIMO based is below in lower SNR area. In Figs. 5 as well as 6 the sum rate for the proposed technique is higher in lower area of SNR. But in higher area the conventional MIMO based channel estimation has the highest sum-rate. As in beamspace channel estimation technique we have to work on low SNR region that is because we won't be worried about this result.

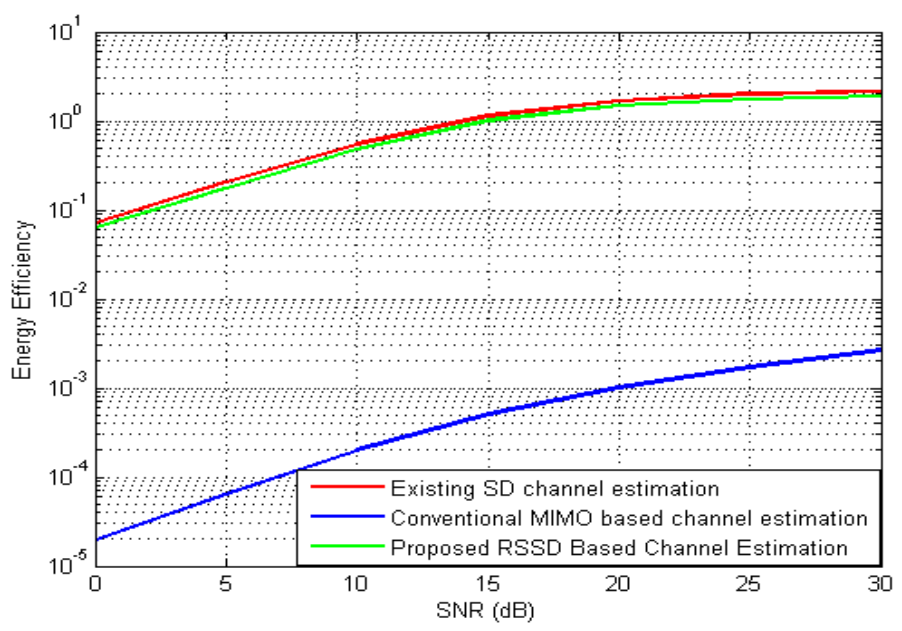

Figure 8. EE for 64 users with LoS effect.

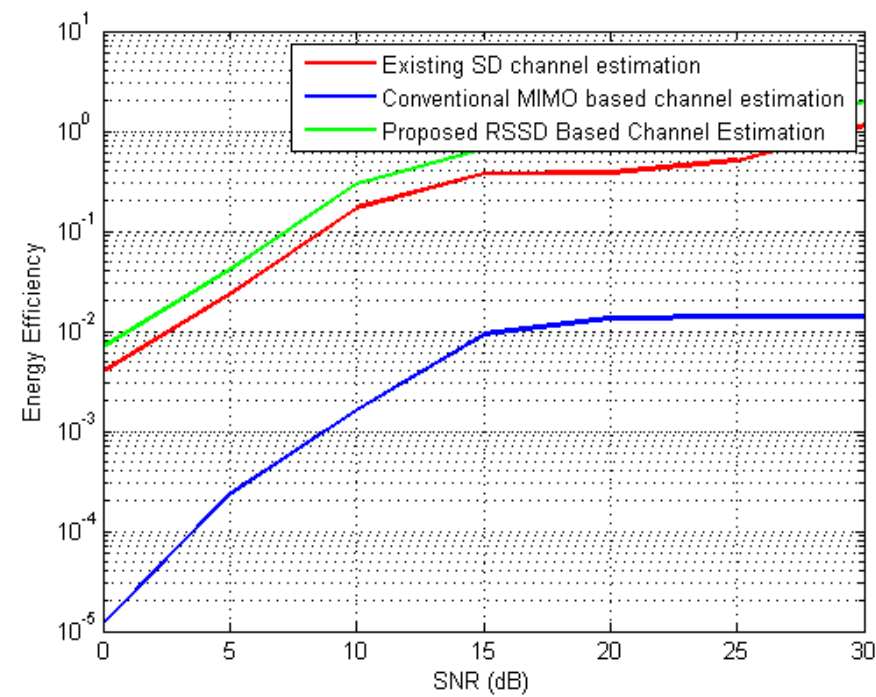

Figure 9. EE for 64 users with NLoS effect. 




Figure 10. EE for 64 users with fading effect.

Fig. 8 represents Energy Efficiency (EE) for the proposed scheme comparing with the conventional MIMO based channel estimation and the existing support detection channel estimation technique. Here energy efficiency vs SNR (dB) graph has been considered. In the Fig. 8, we consider the LoS effect. Here we can observe that the conventional MIMO based channel estimation technique has the low rate of EE in low SNR region. The existing method gives us the EE from $10^{-2}$ for $0 \mathrm{~dB}$ SNR and also increase with the SNR $(\mathrm{dB})$. But in high SNR region the conventional MIMO gives us the better result than the existing method. However, our proposed model gives us the better result in both two SNR areas. In Fig. 9 describes the NLoS effect for the proposed model. Here it is very clear that our proposed mechanism is far better than the other two mechanisms.

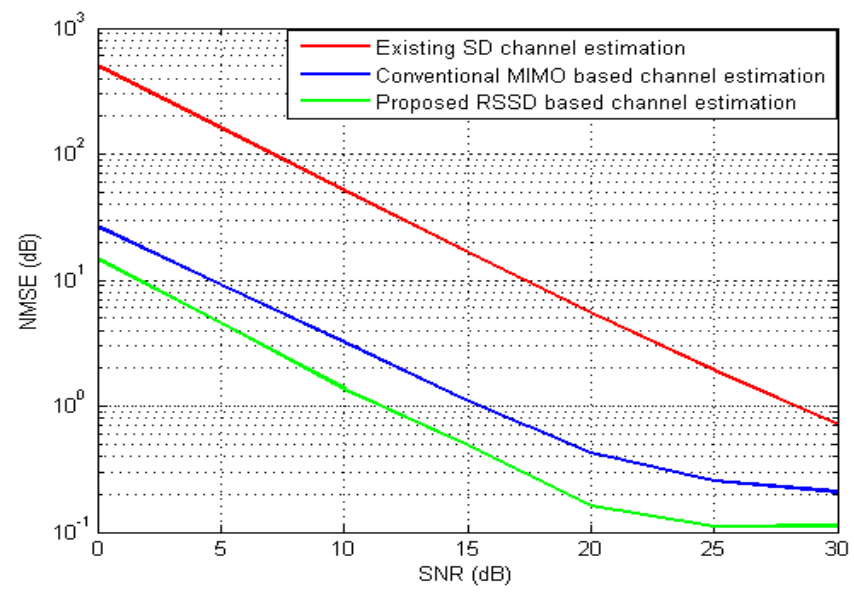

Figure 11. NMSE for LoS effect with 128 users. 
International Journal of Wireless \& Mobile Networks (IJWMN) Vol. 13, No.1, February 2021

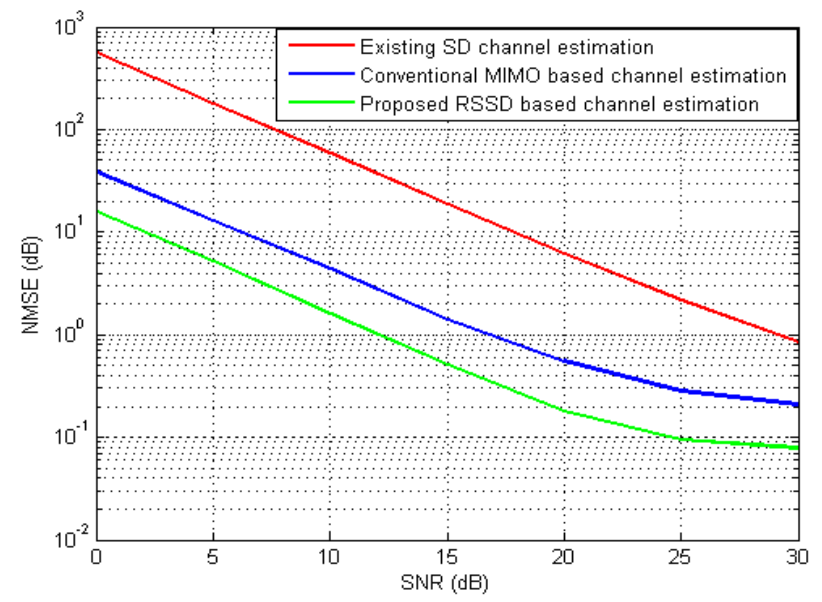

Figure 12. NMSE for NLoS effect with 128 users.

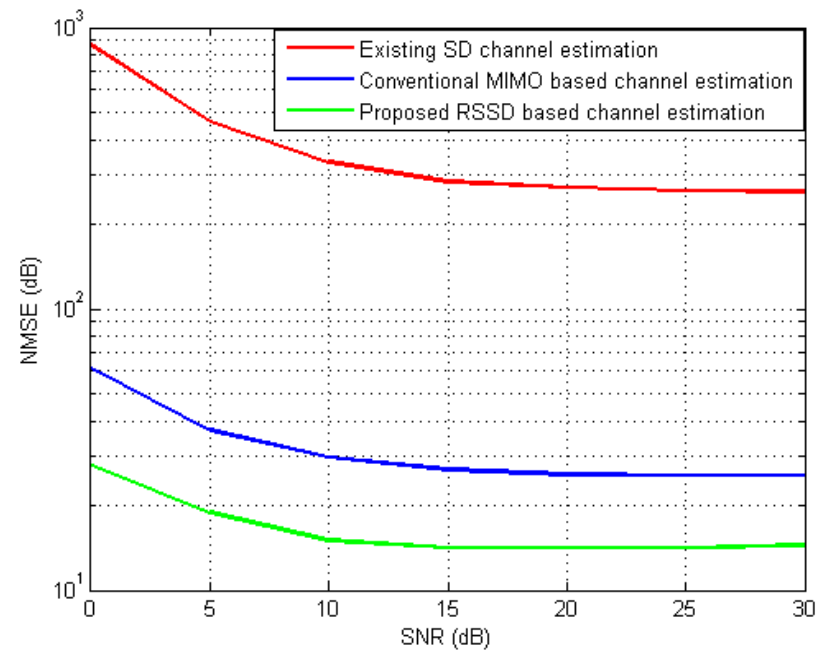

Figure 13. NMSE for fading effect with 128 users.

Fig. 10 sketches the fading effect. Here the proposed scheme is far better than the existing SDbased channel estimation technique. But EE shows slightly lower result in both two areas. 
International Journal of Wireless \& Mobile Networks (IJWMN) Vol. 13, No.1, February 2021

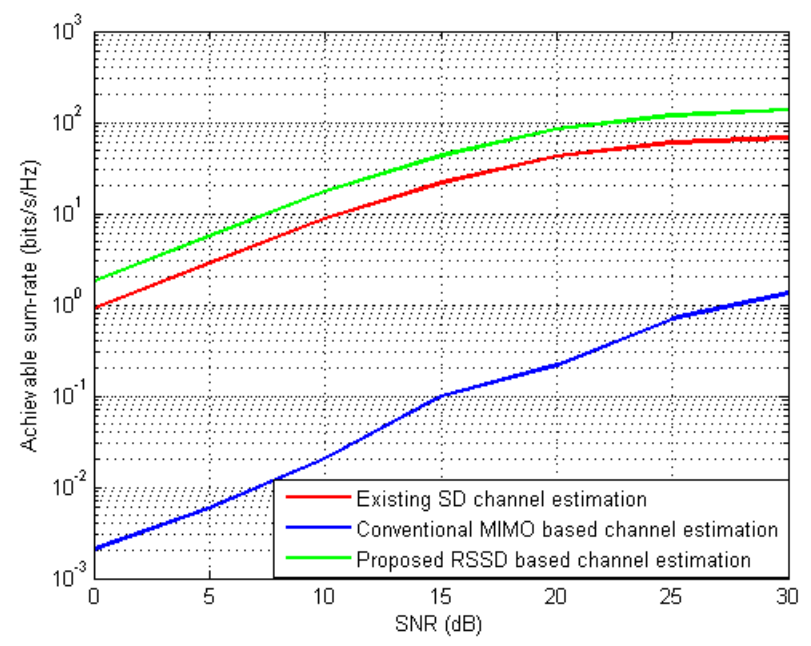

Figure 14. SE for 128 users with LoS effect.

Figs. 11, 12, 13 and Figs. 14, 15, 16 as well as Figs. 17, 18, 19 describe the NMSE, achievable sum-rate (SE) and EE respectively, for 128 users.

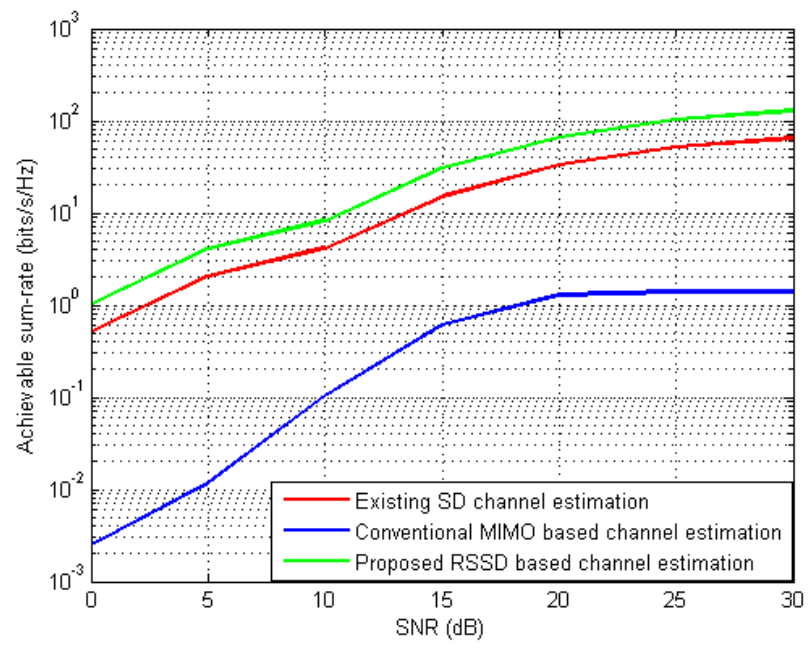

Figure 15. SE for 128 users with NLoS effect. 
International Journal of Wireless \& Mobile Networks (IJWMN) Vol. 13, No.1, February 2021

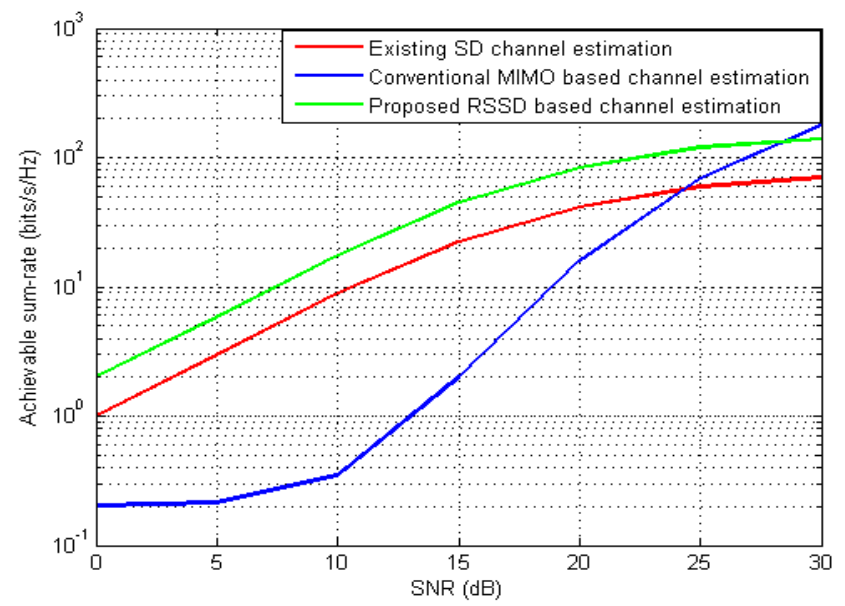

Figure 16. SE for 128 users with fading effect.

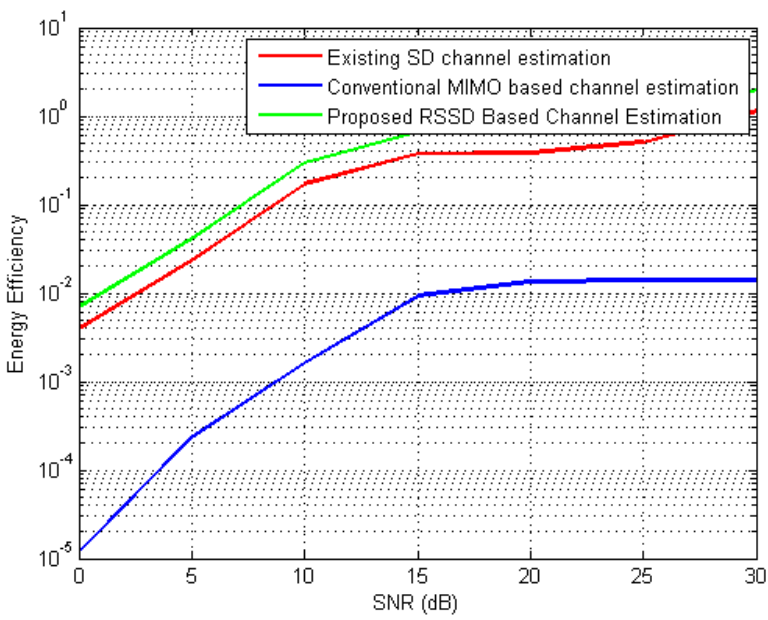

Figure 17. EE for 128 users with LoS effect.

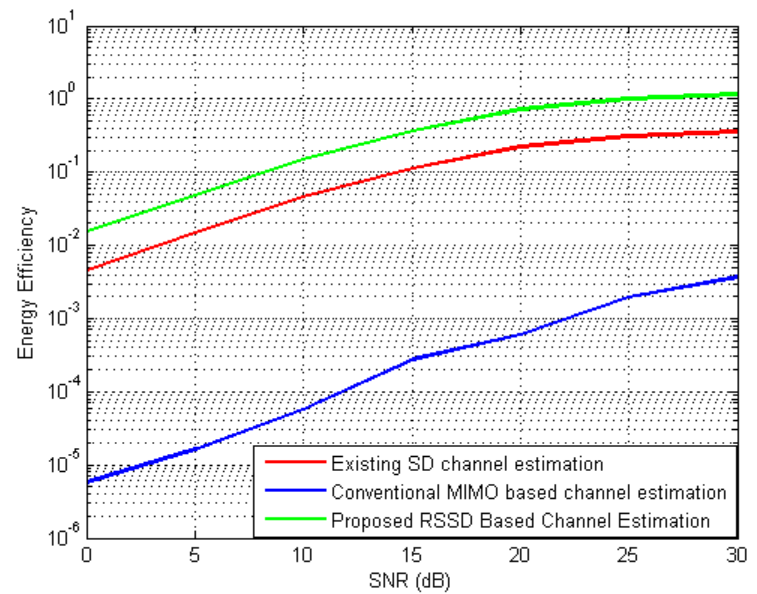

Figure 18. EE for 128 users with NLoS effect. 


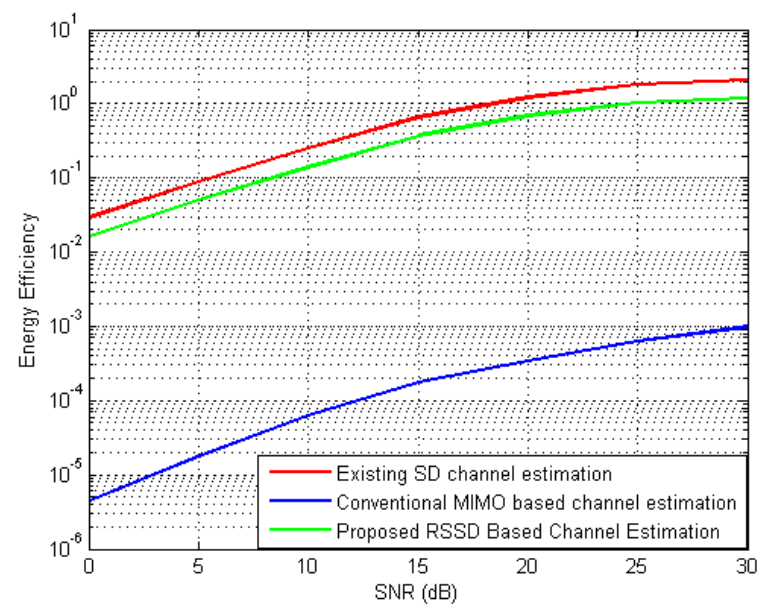

Figure 19. EE for 128 users with fading effect.

Here we can observe that proposed mechanism experiences us the better result comparing with other two except the Fig. 19. In Fig. 19 we observe that the EE for fading effect of the existing methods result the better output than the proposed method but we get higher EE than the conventional MIMO based channel estimation technique.

\section{ConClusions}

In this paper, we present a beamspace channel estimation technique containing a 3D metallic plate lens antenna array. We numerically analysis the beamspace channel. The proposed design includes a model using 3D lens antenna array with voltage doubler and a component selecting procedure which reduces cost. After that a switch is used for detecting the beam. At the end, using the concept of detecting the channel component according to a column, the channel is estimated which enhances system performances mostly such as spectral efficiency and energy efficiency. We simulate the proposed model with various combination of network scenarios such as low user, high users, LoS, NLoS etc. Simulation results exhibit the better performance of the proposed mechanism comparing with the existing conventional MIMO based channel estimation and SD based channel estimation techniques, specifically at the lower SNR area. Future research will explore different channel environments with upcoming beyond $5 \mathrm{G}$ network scenarios.

\section{REFERENCES}

[1] M. M. Mowla, I. Ahmad, D. Habibi and Q. V. Phung, "A Green Communication Model for 5G Systems," in 2017 IEEE Transactions on Green Communications and Networking, vol. 1, no. 3, pp. 264-280, doi: 10.1109/TGCN.2017.2700855.

[2] L. Dai, X. Gao, S. Han, I. Chih-Lin, X. Wang, Beamspace channel estimation for millimeter-wave massive MIMO systems with lens antenna array, in: 2016 IEEE/CIC International Conference on Communications in China (ICCC), IEEE, 2016, pp. 1-6.

[3] J. Brady, N. Behdad, A. M. Sayeed, Beamspace MIMO for millimeter-wave communications: System architecture, modeling, analysis, and measurements, IEEE Transactions on Antennas and Propagation 61 (7) (2013) 3814-3827.

[4] A. Sayeed, J. Brady, Beamspace MIMO for high-dimensional multiuser communication at millimeter-wave frequencies, in: 2013 IEEE global communications conference (GLOBECOM), IEEE, 2013, pp. 3679-3684.

[5] Q. Duan, T. Kim, H. Huang, K. Liu, G. Wang, Aod and aoa tracking with directional sounding beam design for millimeter wave MIMO systems, in: 2015 IEEE 26th Annual International 
International Journal of Wireless \& Mobile Networks (IJWMN) Vol. 13, No.1, February 2021

Symposium on Personal, Indoor, and Mobile Radio Communications (PIMRC), IEEE, 2015, pp. 2271-2276.

[6] X. Gao, L. Dai, Y. Sun, S. Han, I. Chih-Lin, Machine learning inspired energy-efficient hybrid precoding for mmWave massive MIMO systems, in: 2017 IEEE International Conference on Communications (ICC), IEEE, 2017, pp. 1-6.

[7] P. Paul, M. M. Mowla, A novel beamspace channel estimation technique for millimeter wave massive MIMO systems, in: 2019 3rd International Conference on Electrical, Computer \& Telecommunication Engineering (ICECTE), IEEE, 2019, pp. 185-188.

[8] Y. Liu, Q. Feng, Q. Wu, Y. Zhang, M. Jin, T. Qiu, Energy-efficient hybrid precoding with low complexity for mmWave massive MIMO systems, IEEE Access 7 (2019) 95009-95020.

[9] Y. Zeng, R. Zhang, Millimeter wave MIMO with lens antenna array: A new path division multiplexing paradigm, IEEE Transactions on Communications 64 (4) (2016) 1557-1571

[10] M. Nazzal, M. A. Aygu“1, A. G*or cin, H. Arslan, Dictionary learning-based beamspace channel estimation in millimeter-wave massive MIMO systems with a lens antenna array, in: 2019 15th International Wireless Communications \& Mobile Computing Conference (IWCMC), IEEE, 2019, pp. 20-25.

[11] L. Dai, B. Wang, M. Peng, S. Chen, Hybrid precoding-based millimetre wave massive MIMOnoma with simultaneous wireless information and power transfer, IEEE Journal on Selected Areas in Communications 37 (1) (2018) 131-141

[12] T. S. Rappaport, J. N. Murdock, F. Gutierrez, State of the art in 60-ghz integrated circuits and systems for wireless communications, Proceedings of the IEEE 99 (8) (2011) 1390-1436.

[13] A. Reyna, M. Panduro, Optimization of a scannable pattern for uniform planar antenna arrays to minimize the side lobe level, Journal of Electromagnetic Waves and Applications 22 (16) (2008) 2241-2250.

[14] T. Cheng, Y. Song, T. Li, F. Li, H. Liu, Low-complexity channel estimation in 3d lens millimeterwave massive MIMO systems, in: 2018 24th Asia Pacific Conference on Communications (APCC), IEEE, 2018, pp. 347-352.

[15] X. Gao, L. Dai, S. Han, I. Chih-Lin, F. Adachi, Beamspace channel estimation for 3d lens-based millimeter-wave massive MIMO systems, in: $20168^{\text {th }}$ International Conference on Wireless Communications \& Signal Processing (WCSP), IEEE, 2016, pp. 1-5

[16] Tsinghua university, publication. [link]. http://oa.ee.tsinghua.edu.cn/dailinglong/publications/

[17] Z. Xiao, T. He, P. Xia, X.-G. Xia, Hierarchical codebook design for beamforming training in millimeter-wave communication, IEEE Transactions on Wireless Communications 15 (5) (2016) 3380-3392.

[18] X. Gao, L. Dai, S. Han, I. Chih-Lin, R. W. Heath, Energy-efficient hybrid analog and digital precoding for mmWave MIMO systems with large antenna arrays, IEEE Journal on Selected Areas in Communications 34 (4) (2016) 998-1009.

\section{AuTHORS}

Prosenjit Paul is a student of Rajshahi University of Engineering \& Technology (RUET), Rajshahi, Bangladesh. His research interests include 5G, massive MIMO, and millimeter wave.

Md Munjure Mowla received the $\mathrm{PhD}$ degree from the School of Engineering, Edith Cowan University, Australia. His research interests include network planning, optimization, and resource management for small cells heterogeneous network and backhaul network in 5G wireless communication.

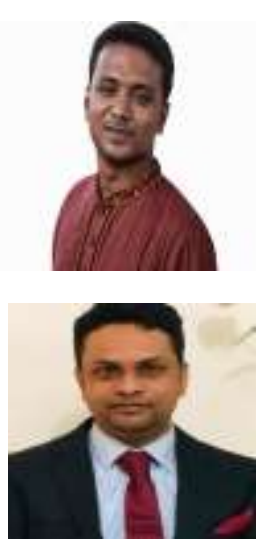

